\title{
AN OVERVIEW OF THE ORBITING SOLAR LABORATORY
}

\author{
D.S. SPICER \\ Laboratory for Astronomy and Solar Physics, NASA/Goddard Space Flight Center
}

\begin{abstract}
The Orbiting Solar Laboratory (OSL), which is a joint NASA/BMFT/ASI/ AFGL program, will be a free flying, polar orbiting complement of five scientific instruments operating as a facility and designed to observe the surface and upper atmosphere of the sun with high spatial and temporal resolution over a spectral range from the soft $\mathrm{X}$-ray to the near Infrared. OSL's primary scientific objectives are to study the coupling of velocity, magnetic, and radiation fields in the photosphere and chromosphere and to causally relate this coupling to activity in the overlying regions.

To study the photosphere and chromosphere with a spatial resolution approaching 75 $\mathrm{km}$ OSL will utilize a 1 meter telescope, optimized for 2000-11,000 $\AA$, to feed a narrow band tunable filter, a set of fixed broad-band filters, and a spectrograph. These three instruments share a common structure, share a unified science data and control system, and use a single-image stabilization system for their common fields of view to form a Coordinated Instrument Package (CIP). To study the transition zone a stigmatic High Resolution Telescope Spectrograph (HRTS) optimized for 1175-1700 $\AA$, will be used capable of providing both images and spectroscopic information. A X-Ray Extreme Ultraviolet Imager and Spectroheliograph (XUVI) optimized for the 40-400 $\AA$ regime, will allow study of the corona and of multimillion degree solar transient events such as flares. Both the HRTS and the XUVI instruments will possess image stabilization systems and have a resolving power of $350 \mathrm{~km}$. All OSL instruments will operate overlapping fields of view and be co-aligned. To achieve its goals the OSL will provide a total data stream of $20 \mathrm{Mbits} / \mathrm{s}$, nominally $4 \mathrm{Mbits} / \mathrm{s}$ per instrument and has a planned operational lifetime of three years.
\end{abstract}

Y. Kondo (ed.), Observatories in Earth Orbit and Beyond, 285.

(C)1990 Kluwer Academic Publishers. Printed. in The Netherlands. 